\title{
Liver resection for concomitant colorectal liver metastases and intrahepatic cholangiocarcinoma: a rare combination
}

\author{
Georgios C. Sotiropoulos • Evangelos Tagkalos • \\ Andreas Kreft • Vasiliy Moskalenko • Ursula Gönner • \\ Ernesto P. Molmenti • Stephan Timm • Theodor Junginger • \\ Hauke Lang
}

Accepted: 2 April 2009/Published online: 25 April 2009

(C) Springer-Verlag 2009

Keywords Liver resection - Colorectal cancer.

Liver metastases $\cdot$ Intrahepatic cholangiocarcinoma

The occurence of simultaneous unrelated tumors has always been of clinical interest. Although the finding of two benign primary liver lesions or of a benign and a malignant one is not infrequent, the co-existence of two unrelated malignant liver tumors is extremely rare. We herein report the case of colorectal liver metastases in association with an intrahepatic cholangiocarcinoma.

A 62-year-old Caucasian female underwent resection of a ypT3 ypN0(0/30) cM0 rectal cancer after receiving neoadjuvant radiochemotherapy. Almost 6 years later, in the setting of tumor markers within normal laboratory range (carcinoembryonic antigen, $<5 \mathrm{ng} / \mathrm{ml}, \mathrm{Ca} 19-9,<37 \mathrm{U} / \mathrm{ml}$ ), a routine follow-up computed tomography (CT) evaluation showed three new liver masses located in segments 2 and 3. The patient was subjected to a non-typical liver resection of the lesions in segments 2 and 3. Pathological exam of the 1.4-

G. C. Sotiropoulos $(\bowtie) \cdot$ E. Tagkalos $\cdot$ V. Moskalenko

U. Gönner $\cdot$ S. Timm $\cdot$ T. Junginger $\cdot H$. Lang

Department of General and Abdominal Surgery,

Johannes Gutenberg University Hospital Mainz,

Langenbeckstrasse 1 ,

55131 Mainz, Germany

e-mail: sotiropoulos@ach.klinik.uni-mainz.de

A. Kreft

Institute of Pathology,

Johannes Gutenberg University Hospital Mainz,

Mainz, Germany

E. P. Molmenti

Department of Surgery, North Shore University Hospital,

Manhasset, NY, USA and 4.6-cm lesions resected from segments 2 and 3 revealed metastatic rectal adenocarcinoma. Immunohistochemichal staining positive for cytokeratin (CK) 20 and CDX2 and negative for CK7 confirmed the diagnosis. The third lesion, $1.9 \mathrm{~cm}$ in size, was found to be a well-differentiated pT1 G1 pR0 cholangiocarcinoma, negative for CK20 and CDX2 and positive for CK7.

Follow-up CT scan of the abdomen 5 months later detected a $0.8-\mathrm{cm}$ cystic mass in segment 7 with no evidence of extra-hepatic disease. An explorative laparotomy was undertaken followed by an atypical resection of segment 7 . Immunohistochemichal staining of the resected lesion, positive for CK20 and negative for CK7, was consistent with metastatic rectal adenocarcinoma. Resection margins were free of tumor. The patient is currently tumor-free, alive, and well 12 months after the third resection and 76 months after the initial operation. Tumor markers remain within normal laboratory range.

To the best of our knowledge, this is the first report of a liver resection for concomitant colorectal liver metastases and intrahepatic cholangiocarcinoma. In the literature, there are only sporadic reports about colorectal metastases with intrabiliary growth, presenting as cholangiocarcinomas of the large ducts, which can be particularly difficult to distinguish morphologically from some cholangiocarcinomas. A combined immunohistochemistry of CK7 and CK20 is useful for this differentiation. In the present case, the different malignant origin of the liver lesions could be well documented in the immunohistochemistry, and a metastatic carcinoma with intrahepatic tumor growth could be excluded in this report, underlying its rarity.

Conflict of interest The authors declare no conflict of interest. 